\title{
Detecting and identifying artificial acoustic emission signals in an industrial fatigue environment
}

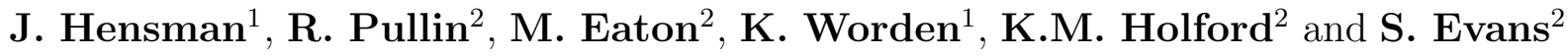 \\ ${ }^{1}$ Department of Mechanical Engineering, The University of Sheffield, Mappin Street, \\ Sheffield S1 3JD, UK. \\ ${ }^{2}$ Cardiff School of Engineering, Cardiff University, Queen's Buildings, The Parade, \\ Cardiff CF24 3AA, Wales, UK
}

\begin{abstract}
This paper details progress towards the application of a methodology for Acoustic Emission (AE) detection and interpretation for the monitoring of fatigue fractures in large-scale industrial environments. An artificial acoustic emission source, representative of a fatigue fracture was injected into a test of a substantial landing gear component. An AE monitoring system was then used to successfully locate and identify the source using the new signal processing methodology.
\end{abstract}

Keywords: Acoustic Emission, crack detection and location, clustering, signal processing.

\section{Introduction}

This paper describes progress in a project to provide an Acoustic Emission (AE) system for monitoring aircraft landing gear for fatigue fractures during long-term testing. The AE approach is a technique for passively 'listening' for ultrasonic activity in a structure, using a network of surface mounted transducers. Damage-related events within the structure release small amounts of energy which propagate as ultrasonic stress waves through the structure, to be detected and interpreted by the AE equipment. AE sensors are typically responsive to surface displacements in the picometer range, and so sparse networks of sensors can be used to monitor large structures at relatively low cost.

The long term tests which aircraft landing gear are subjected to before certification are necessary to certify the structural integrity of the gear. Non-destructive testing is performed periodically to support the integrity demonstration of the structure and this adds significant time to the overall testing regime that can last 2 to 3 years. The current project aims to provide a detection system to support the removal of periodic NDT and an 'early warning' system that detects the presence of fatigue fracture AE before rupture, so that necessary steps can be taken.

During a fatigue test, the AE monitoring system will detect not only AE activity related to fatigue fracture growth, but also activity from fretting and rubbing of components and other spurious sources; in fact, the overwhelming majority of detected acoustic activity will be the result of common processes unrelated to crack propagation. In this paper, an AE system is configured to monitor a significant component of an A340 landing gear during routine testing, and an artificial AE source is added to represent a fracture. A methodology is presented which can be used to detect the artificial source amongst the huge number of unrelated AE signals. 


\section{Methodology}

The methodology for the AE identification strategy evaluated here is carried-out in three main phases, so this section of the paper is divided into three parts: The first part details the methods used to ensure that the artificial AE source was representative of a fatigue fracture; this is followed by details of the fatigue test machinery and AE recording equipment and the third section describes the signal processing techniques used to deal with the data.

\section{Design of the Input Signal}

The detection of an artificial AE source amongst 'everyday' AE activity would be relatively straightforward if the artificial source were not in some way representative of a fracture. If the artificial source was at a much higher amplitude than the other sources, or was exited in a different frequency band, the task of identifying it would be trivial. In order to make the task of identifying the artificial source non-trivial and representative of reality, the signal input to the transmitting transducer was carefully selected to faithfully represent a fatigue fracture.

The transmitting transducer chosen was a Physical Acoustic Pico, with a resonant frequency of $500 \mathrm{kHz}$, and operating frequency range of $200-750 \mathrm{kHz}$. This transducer was selected for its response characteristics and also for its convenient small size - the transducer was to be placed in awkward positions on the landing gear. The transducer was attached to a PAL ARB-1410 Arbitrary Waveform Generator board, which periodically excited the Pico transducer with a pre-defined signal, generating artificial acoustic emissions within the structure.

To ensure that the identification task faithfully represented the detection of a fatigue fracture event, preliminary tests were carried out on a small compact tension (CT) coupon of the same material as the landing gear. One CT coupon was fatigue tested to failure, and a typical waveform relating to crack growth was identified. The selected transmitting transducer was placed on a second CT coupon, and the settings of the signal generator were adjusted until the fatigue fracture signal was suitably simulated. The crack signals have to travel further in the real structure than in the test coupon and therefore suffer greater distortion from dispersion. However, if the artificial source and true crack agree at a given distance (in the CT specimen) then they should agree at greater distances. As the CT specimen is of the same material as the landing gear, the only possible source of discrepancy between the true and artificial crack signals is the fact that the thicknesses were not equal and therefore the waves may propagate on different regions of the dispersion curve. This consideration was not considered important as the methodology detects low within-cluster variance of the crack signals and not individual waveform characteristics.

The signal finally selected to excite the transducer was a two-cycle square wave, amplitude $0.5 \mathrm{~V}$, period $2.5 \mu \mathrm{s}$ 


\section{Test Details}

The component designated for inspection was the bogie beam section of the landing gear from an Airbus A340. This section of the gear attaches the main tube to the wheels; it is approximately $2.4 \mathrm{~m}$ long and $0.3 \mathrm{~m}$ in diameter. The full gear was under test, designed to simulate the working conditions of the gear during take-off and landing. This involved the actuation of 16 hydraulic closed loop load channels controlled using a Moog-FCS ssytem to sequence the test regime representing taxiing, take-off, flight and the landing stages of an aircraft's working life. One cycle (representing one flight) is completed in approximately 180 seconds, and contains a series of 'transitions' of frequency $0.3-1 \mathrm{~Hz}$. The bogie beam, with AE sensors attached is shown in the photographs in Figure 1.
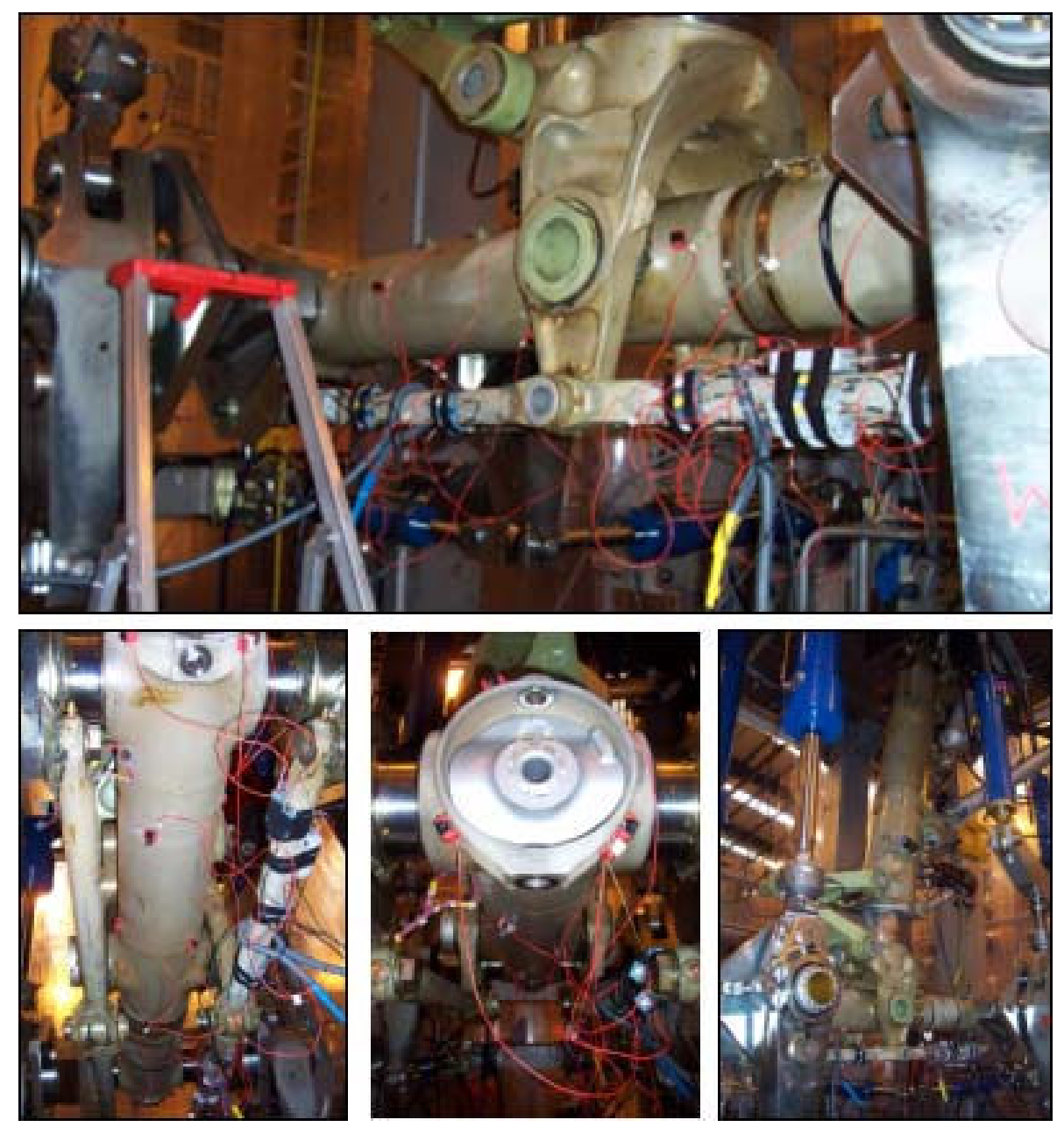

Figure 1: Photographs of the test setup. The bogie beam component is visible in the top photograph; subsequent photographs show the AE sensors attached to the beam by magnetic clamps.

A Physical Acoustics DISP 20 channel system was used to record AE activity during the test. The sensors were distributed according to Figure 2. The sensors used were Physical Acoustics resonant Nano 30 AE sensors, which have a bandwidth of $125-750 \mathrm{kHz}$, and a resonance frequency of $300 \mathrm{kHz}$. Each sensor was connected to an in-line pre-amplifier of gain $40 \mathrm{~dB}$, which also contained anti-aliasing filters. The sample rate used to record 


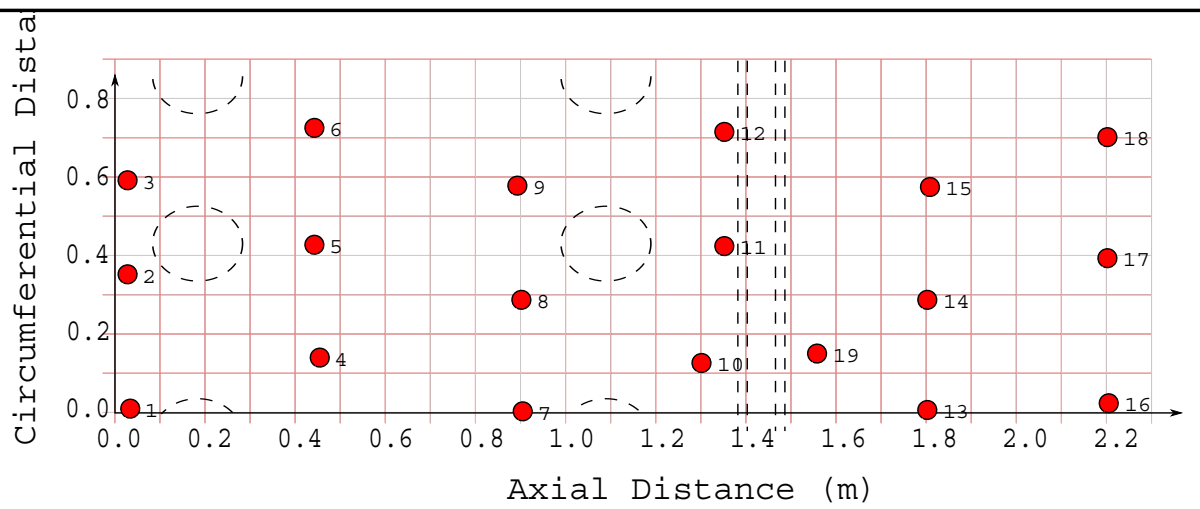

Figure 2: Schematic showing the setup of the AE equipment. The 19 sensors are shown (filled circles), placed on a grid along the bogie beam structure. Structural elements of the beam are identified as dashed lines - vertical lines show a thickness change and dashed circles show connections to other parts.

the AE signals was $40 \mathrm{MHz}$.

A total of three tests were performed over a period of three days; the duration of each test ranged from 1.5 to 3.5 hours, corresponding to approximately 30-70 flight cycles. At the beginning of each test, the artificial AE source was moved to a new position by Messier-Dowty technical staff. These three tests are henceforth denoted Experiment A, B and $\mathrm{C}$. The technicians did not reveal the positions of the sources until the analysis had been carried out. It became clear post-analysis, that there was an anomaly in the fixing condition for the sensor for experiment A, so only experiments B and C are discussed here. the reader can consult [2] for a discussion of experiment A.

In order to properly correspond to the behaviour of a growing fatigue crack, the rate of input of the actuation signals was gradually increased over the duration of the test. This information was not used in the detection or identification of the source, but was used later to verify results and in particular, to explain an anomaly which occurred. It was also used to test the sensitivity to differing rates of AE. There is an underlying assumption here that the AE signals from the crack do not change with time for any other reason e.g. that the amplitudes of the waveforms do not change with time. This assumption requires further investigation as changes in the waveform features with time will inevitably broaden the clusters of crack-generated waveforms. For the purposes of the tests here, the time-invariance of the waveforms was assumed.

\section{Signal Processing}

The test data were post-processed using in-house software, built using the Python scripting language and scientific python modules SciPy [3]. This allowed the development of signal processing methodologies which are not currently available as standard in the $\mathrm{AE}$ capture software. The AE capture software 'AE Win' was used to perform basic feature extraction and location of the data. 


\section{Overview}

The simplest unit of AE data is a hit, which consists of the triggering of a single channel, and the subsequent recording of a signal (or waveform). The AEWin software uses a timing parameter, event-definition-time, to group hits into events: hits which arrive on different channels sufficiently closely-spaced in time, and presumed to have occured at one single source are grouped together as an event. Hits which can not be allocated into events are discarded. For the three tests detailed here, the hit-event ratio was approximately $38 \%$, i.e. $38 \%$ of all detected hits could be successfully allocated to events.

Once the data had been allocated into events, the events were further grouped into location-based 'clusters'. An Online Radius-based Clustering Algorithm (ORACAL) was developed for this task, although other algorithms were considered.

The clusters of events were then ranked by the similarity of the hits in each cluster. This was done by taking the Euclidean distance between the 'feature' data for each signal: the usual AE feature parameters were used (amplitude, rise-time, duration, energy etc.). Clusters containing signals with similar features were ranked highly (most likely to represent the artificial source).

\section{The location algorithm}

The locations of each AE event were calculated from the known positions of the sensors and the speed of sound in the structure, which was assumed to be $4700 \mathrm{~ms}^{-1}$ in this work. One could then estimate the position of an event by minimising the objective function $Z$ in Equation 1,

$$
Z=\sum_{i, j} \Delta T_{i, j}-\frac{\left|S_{i}-S_{j}\right|}{c}
$$

where $S_{i}$ represents the position of sensor $i, \Delta T_{i, j}$ represents the measured difference in the time of arrival between sensors $i$ and $j$, and $c$ is the speed of sound in the material. The indices $i, j$ represent all the sensors which are active for the given hit. $Z$ can be easily minimised using the Nelder-Mead simplex algorithm [4].

\section{Clustering by location}

Once AE events had been assigned locations, they were clustered into groups based on this location.

A simple and popular clustering algorithm is the $k$-means clustering algorithm [5]. This involves the association of each data point (or, AE event in the current context) with one of $k$ centers, and the iterative updating of the center positions and data associations. The major drawback of the algorithm is that it requires the prior specification of $k$, the number of clusters.

Radius-based Clustering ALgorithms (RACALs) such as the Quality Threshold (QT) algorithm [6] and the Self-Organising Oscillator Network [7] do not require the prior specification of the number of clusters; they require instead the expected cluster diameter 
d. In the current application it was considered preferable to specify a cluster diameter since it relates to a physical distance; $d$ can be measured across the surface of the bogie beam component.

An Online RACAL (ORACAL) algorithm was developed especially for this task, and has potential applications in various other disciplines where the data to be clustered is sequential - the order of the data is important, and the clustering can be performed in real time. Details of the algorithm can be found in [2]

\section{Cluster Ranking}

The clusters of events were then ranked according to the similarity of the events within them. Clusters which contained a series of signals which were similar were ranked highly, whilst those containing dissimilar signals were assigned a low rank. Care was taken to only compare signals arriving at the same sensor: any similarities or differences between the signals were therefore attributed to the nature of the source of those signal, not to changes in the propagation path.

Since the artificial AE generator provides a highly repeatable source, it was expected that clusters of events containing similar signals would pertain to the artificial source. Inherrent uncertainties in the nature of other AE sources (such as bearing noise or fretting) would lead to clusters of signals with dissimilar signals.

The metric used to measure the similarity of a group of signals was simply the standard deviation of the signal features. These features have evolved over the years amongst AE researchers and practitioners and include such measurements as the amplitude, energy, duration and peak frequency of the signal.

The standard deviations of the signal features within a cluster were normalised to the standard deviation of the whole set to give a 'tightness' score, $t$ given by Equation 2,

$$
t=\frac{\sum_{i}^{N^{c}} \frac{\left(x_{i}^{c}-\bar{x}^{c}\right)^{2}}{N^{c}}}{\sum_{j}^{N} \frac{\left(x_{j}-\bar{x}\right)^{2}}{N}}
$$

where $x_{i}^{c}$ is a member of the cluster, and $x_{j}$ is a member of the set of all recorded signals; $N$ is the total number of points recorded and $N_{c}$ is the number of point in the cluster of interest. Lower $t$ values indicate greater correspondence between signals in a cluster.

\section{Results}

For each test the cluster diameter required by the ORACAL algorithm was set to $10 \mathrm{~mm}$. This generated hundreds of clusters for each test, nonetheless the tightness parameter proved to be a strong factor in selecting the cluster relating to the artificial source. 


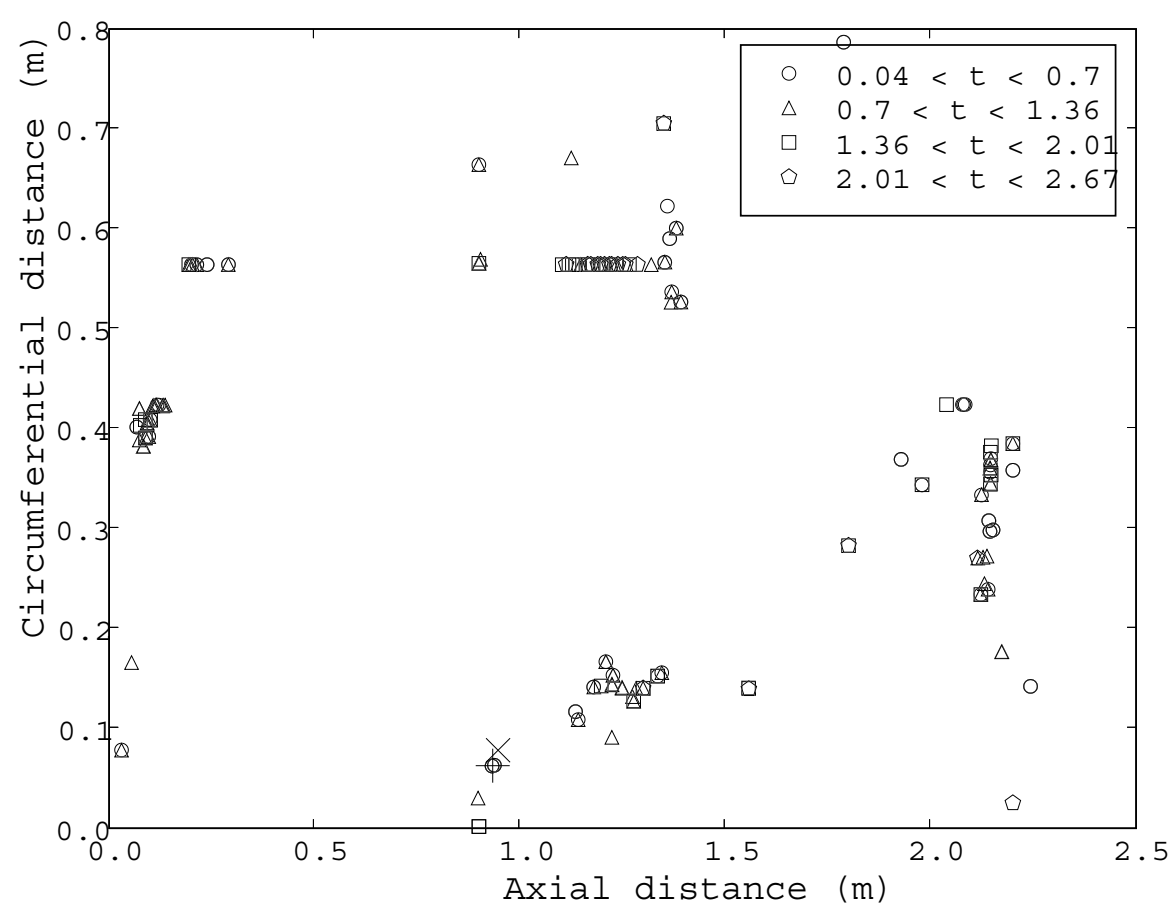

Figure 3: Scatter plot of the clusters detected during experiment B; the symbols show the relative 'tightness' of each cluster. The tightest cluster is shown by the vertical cross at position $(0.93,0.087)$, and the true position of the artificial source is shown by the diagnonal cross at position $(0.95,0.09)$

\section{Experiment B}

Figure 3 shows a scatter plot of the significant clusters found during the second test. The primary cluster is marked with a vertical cross at $(0.93,0.087)$; the true position (diagonal cross) of the artificial AE source was recorded to be $(0.95,0.09), 20 \mathrm{~mm}$ away. The top ranked cluster had a tightness score of 0.04 . This small value and the verification of the position of the source gives some degree of confidence that the detected cluster was the correct one.

In order to verify this, the event rate for the cluster was plotted against the input signal rate in Figure 4. There is a clear correspondence between the two rates, though the detected rate is slightly lower than the known input rate. This is likely due to the small cluster diameter used - using a broader cluster diameter would ensnare signals from a slightly wider area, and would likely correct this minor discrepancy. However, expanding the cluster size would open up the possibility of engulfing events in the cluster which did not relate to the artificial source (making the detected event rate higher than the true one).

The good correlation of the event rates along with the very small tightness value and correct identification of the position are good indicators that the artificial source was correctly identified in this test. 


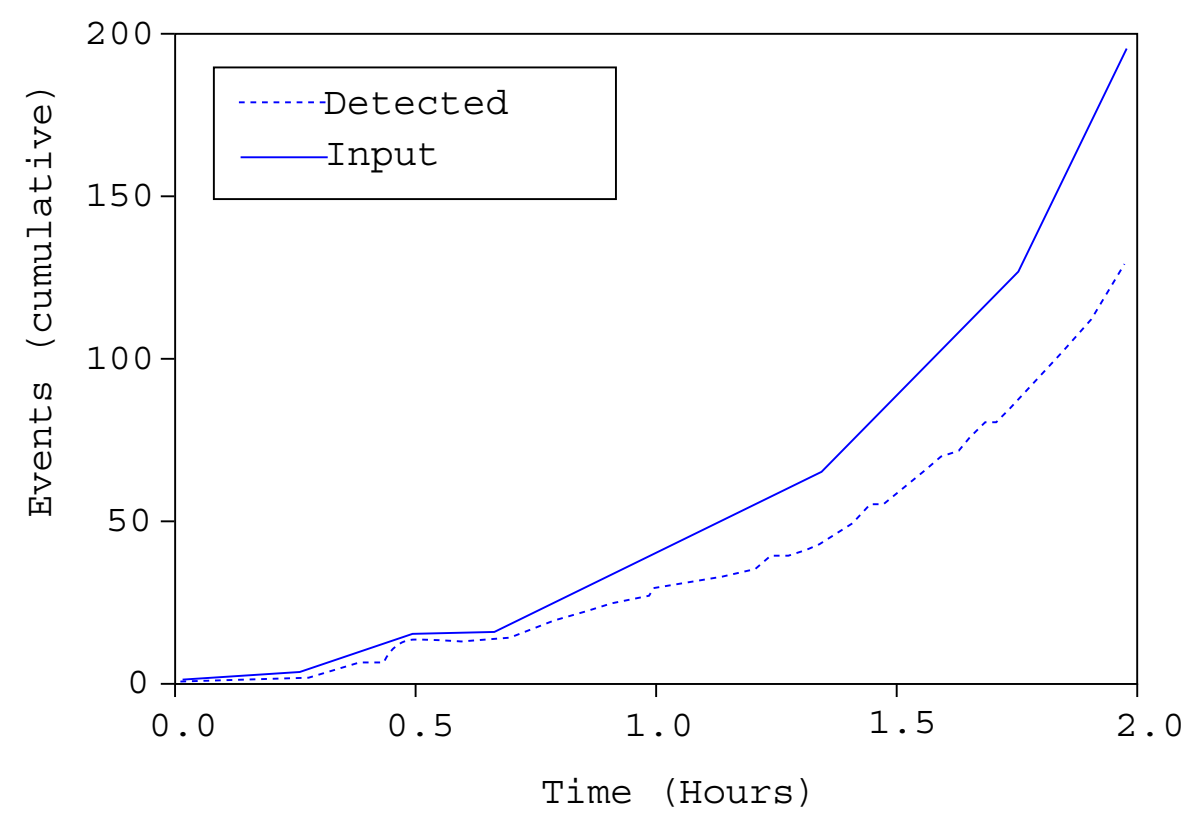

Figure 4: Event rate plot for experiment B, showing the input event rate and the event rate seen in the tightest cluster. The two rates show good correlation, indicating that this cluster does represent data from the artificial source.

\section{Experiment $\mathrm{C}$}

The artificial source was moved again and a third set of data were recorded. The tightest, top-ranked cluster was located at $(1.97,0.34)$, which is $30 \mathrm{~mm}$ from the known position of the source at $(1.95,0.42)$ (the clusters are shown in Figure 5). Again, the tightness score for the top cluster is relatively small - 0.16 - giving a high degree of confidence that the artificial source is represented. Verification comes from Figure 6, where the rate of input artificial AE events is compared to the rate of detected events; an almost perfect correlation is observed.

The near-perfect correlation shown in Figure 6 gives a high degree of confidence that the artificial source was detected. Further, it would seem that these signals were detected with only small loss ( $96 \%$ of transmitted events were detected), and with no confusion with other signals.

\section{Discussion and Conclusions}

This work shows clearly that the developed methodology allows fracture-like signals to be detected in the extremely noisy environment of a structural fatigue test. This is potentially very useful in the sense that it will allow suspension of the test in order to carry out an NDE inspection of the structure in order to evaluate the damage in situ before rupture occurs. The location information provided by the methodology means that the NDE inspection can be immediately directed to the area of interest.

The methodology differs in one important respect from previously developed approaches. 


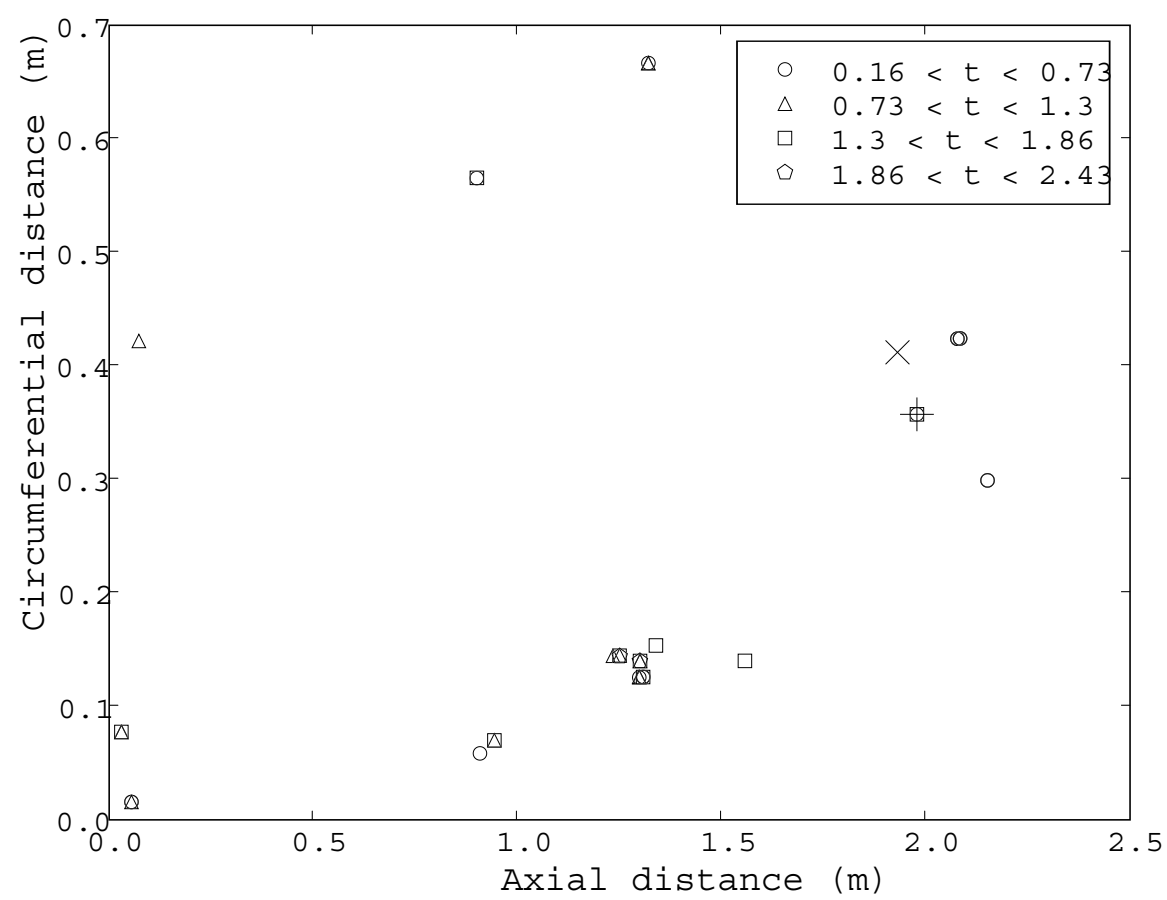

Figure 5: Scatter plot of the clusters detected during experiment C; the symbols show the relative 'tightness' of each cluster. The tightest cluster is shown at position $(1.97,0.34)$ as a vertical cross, and the true position of the artificial source is shown as a diagonal cross at position $(1.95,0.42)$

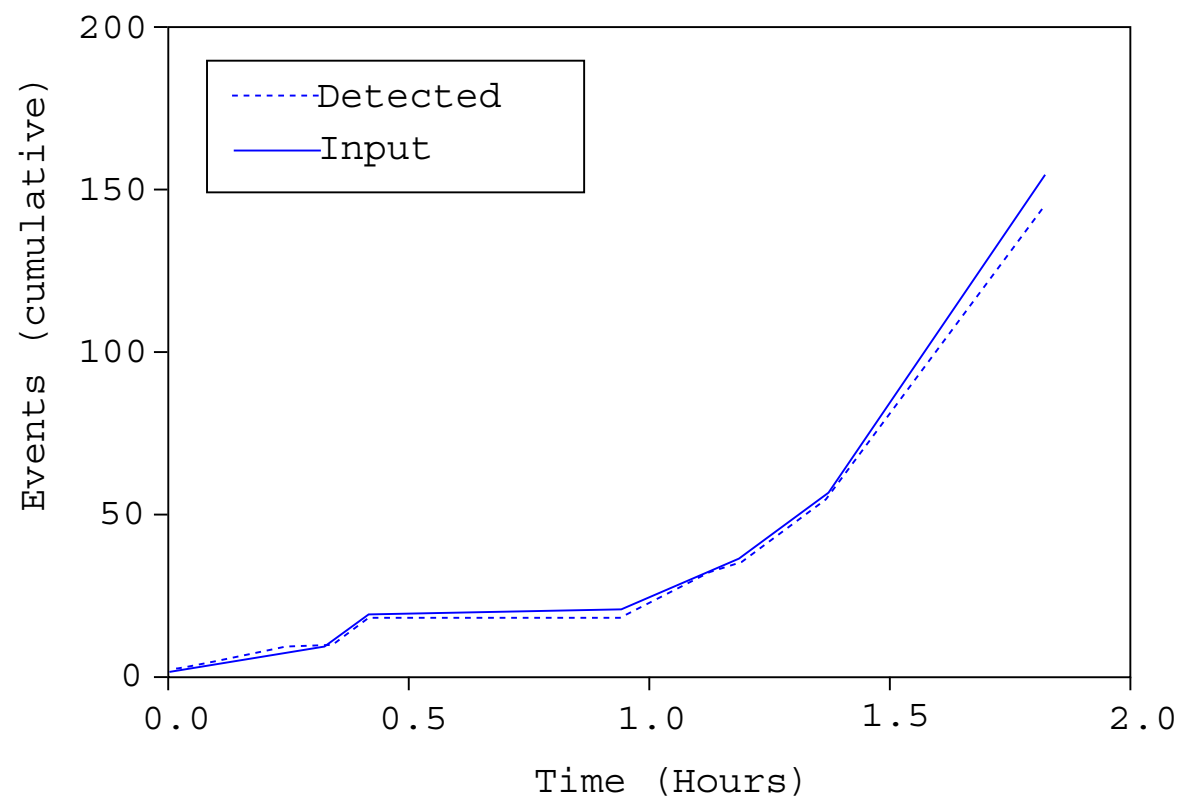

Figure 6: Event rate plot for experiment C, showing the input event rate and the event rate seen in the tightest cluster. The two rates show good correlation, indicating that this cluster does represent data from the artificial source. 
The method does not attempt to recognise the individual burst waveforms characteristic of a crack-propagation event. Rather, the method exploits the fact that crack-related events (propagation at least - not crack-face rubbing) will be very similar to each other and this similarity can be detected by assessing the tightness of the event data when considered in the feature space. The major advantage of this approach is that it is expected to transfer between different materials without difficulty. In contrast, a system that has been carefully trained to recognise crack propagation in Aluminium, will probably not transfer successfully between Aluminium alloys, let alone between different metals.

One of the assumptions inherent in the adoption of this methodology is that the waveform features do not vary significantly with the length of the crack. Such behaviour would cause the clusters of crack-generated events to broaden with time and potentially lose the property of low within-cluster variance. The large-scale test results here support this assumption; however, it is a task for further research to conclusively establish the stationarity of the waveform features.

\section{Acknowledgements}

The authors would like to thank Messier-Dowty Ltd for their financial and technical help in this work, which was also supported by EPSRC grant number GR/T26740/01. The authors would also like to thank the referees of an early draft of the paper for their useful comments.

\section{References}

[1] C.B. Scruby, G.R. Baldwin \& K.A. Stacey, 'Characterisation of fatigue crack extension by quantitative acoustic emission'. International Journal of Fracture 281985.

[2] J. Hensman, R. Pullin, M. Eaton, K. Worden, K.M. Holford \& S. Evans 'Detecting and identifying artificial acoustic emission signals in an industrial fatigue environment'. Submitted to Measurement Science and Technology.

[3] http://www.scipy.org, accessed 15.5.2008.

[4] J.A. Nelder \& R. Mead, 'A simplex method for function minimization'. The Computer Journal 1965.

[5] C.M. Bishop Neural Networks for Pattern Recognition. Oxford University Press, 1995.

[6] L.J. Heyer, S. Kruglyak, S. Yooseph, 'Exploring Expression Data: Identification and Analysis of Coexpressed Genes'. Genome Research 1999.

[7] M.B.H. Rhouma \& H. Frigui, 'Self-organization of pulse-coupled oscillators with application to clustering'. IEEE Transactions on Pattern Analysis and Machine Learning 2001.

[8] B. W. Silverman Density Estimation for Statistics and Data Analysis. Chapman Hall 1985. 